\title{
ATENUACIÓN Y POSIBILIDAD: INTERACCIONES ENTRE DOS CONTENIDOS PROCEDIMENTALES*
}

\author{
Catalina Fuentes Rodríguez \\ Universidad de Sevilla \\ cfuentes@us.es
}

\begin{abstract}
Resumen: El estudio de la atenuación implica tener en cuenta el contenido léxicosemántico de las expresiones, las construcciones en las que aparecen y la presencia de operadores especializados. El presente trabajo analiza dos construcciones, en la medida de lo posible y en lo posible, describe sus usos y los contenidos procedimentales que expresan, que van en dos direcciones: la limitación de la referencia (función marco, argumentativa, ligada al concepto de posibilidad) y la atenuación discursiva (estrategia a la que sirven cuando la minimización afecta a la fuerza ilocutiva). Además, se plantea el grado de fijación de ambas construcciones para determinar si pueden ser consideradas ya operadores discursivos.
\end{abstract}

Palabras clave: operadores discursivos, atenuación, posibilidad, gramaticalización

\begin{abstract}
The study of attenuation implies taking into account the lexical-semantic content of the expressions, the constructions in which appear and the presence of specialized operators. This paper analyzes two constructions, en lo posible and en la medida de lo posible, describes their uses and the procedural meanings they convey, which goes in two directions: the limitation of the reference (argumentative framework function, linked to the concept of possibility) and the discursive attenuation (strategy they serve when minimization affects the illocutive force). The degree of fixation of both constructions is also discussed in order to determine whether they can already be considered discursive operators.
\end{abstract}

Keywords: discourse markers, mitigation, possibility, grammaticalization

"Este trabajo se ha realizado en el marco del Proyecto I+D+I. FFI 2017-82898P «De construcciones periféricas a operadores discursivos: un estudio macrosintáctico del español actual», cofinanciado por el Ministerio de Economía y Competitividad y fondos FEDER. 


\section{INTRODUCCIÓN}

El estudio de la atenuación implica tener en cuenta el contenido léxicosemántico de las unidades, las construcciones en las que aparecen y la presencia de operadores especializados. En cuanto a su campo de acción, incluye la minimización del contenido referencial o de la fuerza ilocutiva del acto de habla. Afecta a la relación entre los participantes del acto y puede emplearse como estrategia argumentativa y con efecto (des)cortés. Algunos operadores o construcciones que van adquiriendo ese valor, como en la medida de lo posible o en lo posible, son atenuativos solo en uno de sus contextos de aparición, si bien el desarrollo de la fijación de este contenido procedimental implica la relación con el contenido epistémico de posibilidad, inserto en su base léxica.

Este trabajo se plantea las siguientes preguntas de investigación: ¿̨cuál es la relación entre la función atenuativa de estas construcciones y su contenido modal?, ¿̨las expresiones indicadas están gramaticalizadas como operadores discursivos? Nuestro estudio tiene, pues, dos direcciones: la discusión teórico-metodológica sobre la relación entre estrategia atenuativa y modalidad y el análisis de posibles operadores discursivos desde el punto de vista de la gramaticalización.

En lo posible y en la medida de lo posible serán descritos en su diversidad funcional, determinaremos sus contextos de realización y comentaremos las relaciones entre los contenidos procedimentales expresados y las estrategias discursivas a las que sirven. Asimismo, relacionaremos estas expresiones con otras que comparten semantismo con ellas. El objetivo es ver, a través del proceso creativo de un operador discursivo, las interacciones que la atenuación establece con otros contenidos macroestructurales en el discurso. La metodología empleada es la Lingüística pragmática (Fuentes Rodríguez, 2017a [2000]).

\section{LA ATENUACIÓN}

La atenuación ${ }^{1}$ ha sido tratada en la bibliografía de forma profusa en los últimos tiempos, sobre todo. Para Briz (1998: 109) es una categoría pragmática surgida de la relación interlocutiva entre hablante y oyente y marcada por el realce de este último. Al mismo tiempo la considera una estrategia (p. 110). La intensificación,

$1 \quad$ Para el estudio de la atenuación consúltese, entre otros, Fraser (1980, 2010), Montes Giraldo (1980-81), Meyer-Hermann (1988), Haverkate (1994), Briz (1995, 1998, 2005 y 2007), Puga Larraín (1997), Caffi (1999 y 2007), Sbisá (2001), Félix-Brasdefer (2004), Douglas de Sirgo (2007), Jørgensen (2009), Kaltenböck-Mihatsch-Schneider (eds. 2010), Fuentes Rodríguez (2010), Albelda y Cestero (2011), Samper Hernández (2011), Cestero y Albelda (2012) y Schneider (2013). 
por su parte, implica un realce de la producción del hablante. En la actualidad Briz y Albelda dirigen un proyecto sobre atenuación y la definen como categoría y estrategia a la vez:

La atenuación es una categoría pragmática en tanto mecanismo estratégico y táctico (por tanto, intencional), que tiene que ver con la efectividad y la eficacia del discurso, con el logro de los fines en la interacción, además de tratarse de una función solo determinable contextualmente.

Es una estrategia, puesto que se atenúa, argumentativamente hablando, para lograr el acuerdo o aceptación del otro (incluso, cuando esta sea solo una aceptación social). Luego, es un mecanismo retórico para convencer, lograr un beneficio, persuadir y, a la vez, para cuidar las relaciones interpersonales y sociales o evitar que estas sufran algún tipo de menoscabo (Briz y Albelda, 2013: 292-3).

Las palabras más repetidas para caracterizar la atenuación son mitigación (Caffi, 1999, 2007) o minimización.

Más concretamente, dicha estrategia consiste lingüísticamente en minorar, minimizar, mitigar, debilitar la acción e intención o el efecto que estas puedan tener o haber tenido en la interacción, y en dicha estrategia están implicados los hablantes, los oyentes e, incluso, terceros (presentes o ausentes). En particular, con tal minoración se logran diversos grados de distanciamiento del mensaje, bien mediante la relativización o bien a través de la impersonalización, que son, de forma abstracta, las tácticas empleadas para atenuar, las cuales se realizan mediante diferentes recursos verbales y no verbales. (Briz y Albelda, 2013: 293)

De este modo se pueden conseguir efectos sociales de acercamiento al oyente y a la vez de distanciamiento lingüístico del mensaje: «lingüísticamente, atenuación significa distancia; socialmente, atenuación significa acercamiento» (Briz-Albelda, 2013: 293).

En el caso que nos ocupa encontramos un procedimiento atenuativo: el uso de la modalidad epistémica para reducir la fuerza de lo dicho. La atenuación es el efecto pragmático conseguido, la estrategia para la que se usan operadores discursivos cuyo contenido apunta a la posibilidad, contenido modal en la bibliografía al uso.

Aunque en la lengua contamos con operadores de posibilidad (posiblemente, quizás, tal vez, verbos como puede...) vamos a reflexionar en este trabajo sobre otras

2 Necesitaría precisarse en qué sentido se habla de « categorías pragmáticas». 
construcciones que pueden ir camino de la fijación y que incluyen el término posible en su base léxica: en lo posible, en la medida de lo posible $e^{3}$. Determinar su puesto en el paradigma, su uso como atenuativo y perfilar su contenido procedimental es uno de los objetivos de este artículo. El otro es, como dijimos más arriba, determinar el grado de fijación de la construcción y preguntarse por su posible consideración como operador $^{4}$ (Fuentes Rodríguez, 2003, 2018[2009]).

\section{EN LO POSIBLE, EN LA MEDIDA DE LO POSIBLE}

Estas dos construcciones, sintagmas con preposición en su origen, mantienen su contenido primario en unos contextos, pero parecen ir adquiriendo valores procedimentales en otros. El adjetivo posible aparece en el primer caso sustantivado, como núcleo de la construcción (en lo posible); en el segundo modifica a medida. Analizamos a continuación el valor de ambas construcciones partiendo de los corpus CREA, CORPES, CORDE y MEsA ${ }^{5}$ y nos planteamos si están ya gramaticalizados como operadores discursivos (Fuentes Rodríguez, 2003, 2018[2009]), es decir, como elementos con funcionamiento macroestructural que apunta al hablante (enunciación, modalidad) o al oyente (argumentación, información). El proceso incluye diversos pasos: la desemantización (bleaching), la extensión o generalización, la decategorización y la erosión o reducción fonética. Hopper (1991) recoge layering (coexistencia de funciones distintas), divergencia, especialización, persistencia y decategorización. Lehmann (1982: 11) considera que la «gramaticalization is a process which may not only change a lexical item into a grammatical item but also shift an item 'from a less grammatical to a more grammatical status' in Kuryłowicz words [...]; grammaticalization is a process of gradual change». El cambio de lo semántico a lo gramatical puede entenderse como una pérdida de contenido (bleaching) o el desarrollo de un contenido «gramatical». En el caso de los marcadores discursivos se ha aludido al reanálisis o a la pragmaticalización (Erman-Kotsinas, 1993; Dostie, 2004; Claridge-Arnovick, 2010; Diewald, 2011), ya que se trata de

the process by which a lexico-grammatical sequence or word form, in a given context, loses its propositional meaning in favour of an essentially metacom-

\footnotetext{
${ }^{3}$ Relacionadas con ellas pueden estar las construcciones a ser posible, si es posible, que por cuestiones de espacio no analizamos aquí.

${ }^{4}$ En consonancia con los objetivos del proyecto en que se enmarca este trabajo. Véase nota inicial.

${ }^{5}$ El corpus MEsA ha sido elaborado por el grupo de investigación APL y está formado por material procedente del discurso digital: foros, Facebook, Twitter, Whatsapp, blogs, páginas web, Instagram, Youtube. http://grupo.us.es/grupoapl/otrosapartados.php?otro=10.
} 
municative, discourse interactional meaning and/or (an already pragmatic element) continues to develop further pragmatic functions or forms (Claridge-Arnovick 2010: 187).

Este proceso «starts and ends in discourse» (idem), los términos léxicos se usan con funciones ilocutivas o discursivas. Claridge y Arnovick (2010) proponen el término discursisation cuando un elemento pragmático desarrolla funciones discursivas nuevas, usando su multifuncionalidad, algo reconocido por otros autores (Brinton, 2010; Fuentes Rodríguez, 1996).

En las estructuras que estudiamos en este trabajo se produce una desemantización, al menos en uno de los empleos, como propugna Heine (2003: 579), y una generalización del contenido. Este pasa de lo designativo a lo procedimental, como hemos defendido para otras unidades (Fuentes Rodríguez, 2016). En todos ellos se cumple el layering y también el proceso de intersubjetivización (López Couso, 2010), entendido como una referencia al campo del hablante (Fuentes Rodríguez, 2016) ${ }^{6}$. Como Cifuentes Honrubia (2003: 14) afirma, obtenemos un contenido más abstracto: «un aspecto común en todas las definiciones dadas sobre gramaticalización es la adquisición por parte de una unidad lingüística de un contenido gramatical o más abstracto, o bien el paso de una unidad de contenido gramatical a otro más gramatical».

Traugott (2003: 645) lo liga al contexto: «(...) the process whereby lexical material in highly constrained pragmatic and morphosyntactic context is assigned grammatical function, and once grammatical, is assigned increasingly grammatical, operator-like function $\gg$. Algunos autores han defendido un proceso de gramaticalización en el que se impliquen razones o funciones pragmáticas, que pasan, así, a la gramática. Garachana (1999: 156-7) propone el proceso siguiente para encima: sintagma preposicional > adverbio > conector aditivo > interjección impropia.

En Fuentes Rodríguez (2016) proponemos para para colmo un cambio como el que reinvidican Traugott (1995) o Company (2003): adverbios o sintagmas preposicionales que pasan a desarrollar una función como marcador discursivo, sea este conector u operador (Fuentes Rodríguez, 2003). El proceso sufrido es una fijación sintáctica, generalmente una extraproposicionalidad sintáctica (Dik, 1997; Fuentes Rodríguez, 2007), un salto a la macrosintaxis (Berrendonner, 1990, 2003; Blanche Benveniste, 2002; Fuentes Rodríguez, 2017b), y semánticamente un debilitamiento de contenido que lo lleva a lo procedimental, al desarrollo de contenidos macroestructurales, dependientes del entorno. Los que separan gramática y pragmática los

${ }^{6}$ Consúltese también Traugott (1995), Stein-Wright (1995) o Nuyts (2001). 
consideran contenidos pragmáticos ${ }^{7}$. Como Company (2003: 14) defiende, el proceso consiste más bien en una generalización del contenido, un proceso

[...] simultáneo de debilitamiento o pérdida y ganancia: pérdida de especificidades de significado y de privilegios de ocurrencia, y debilitamiento de iconicidad, pero al mismo tiempo es ganancia en polisemia, incremento de funciones adicionales gramaticales y aumento en la frecuencia y generalización del signo, y por lo tanto ganancia en la aplicabilidad del signo.

A ello acompaña normalmente una pérdida de sustancia fónica y la fusión de sus componentes ${ }^{8}$.

Heine (2013: 1205-6) propone hablar, en el marco de su thetical grammar (Kaltenböck-Heine-Kuteva, 2011), de un proceso previo de cooptation, que consiste en que «units such as clauses, phrases, or words are taken from the domain of sentence grammar and deployed for purposes of discourse organization $\gg$. Pasemos a comprobarlo en el análisis.

\subsection{En lo posible}

3.1.1. Esta estructura aparece como una construcción fijada, no admite inserción de elementos: *en lo muy posible, *en lo más posible. Es un sintagma en origen que actúa como grupo, con una función y significado únicos. Cumple, así, uno de los pasos de la gramaticalización. Tenemos que preguntarnos si su contenido deriva del de sus miembros o ha sufrido un proceso de debilitamiento o generalización.

La base léxica es el adjetivo posible, con lo que deriva al campo de la modalidad epistémica. Con ella el hablante presenta los hechos de manera que no puede asertar su verdad o falsedad. Generalmente esto ocurre con entornos de futuro o modalmente marcados. Sin embargo, tenemos que decir que la construcción que estudiamos aquí no parece relacionarse de forma prototípica con el concepto de posibilidad. Si comparamos las siguientes realizaciones podremos ver las diferencias:

(1a) Posiblemente me matricule en el máster el próximo año.

${ }^{7}$ Y por ello consideran marcadores discursivos a conjunciones como $y$ o pero cuando relacionan enunciados o intervenciones. ¿Su categoría ha cambiado? Se identifica, así, la gramática con la sintaxis de la oración, una limitación que, como hemos indicado en otros lugares, no suscribimos (Fuentes Rodríguez, 2017b).

${ }^{8}$ Cfr., al respecto, también Buenafuentes (2007). 
Indica acción futura que no se afirma como verdadera o falsa. En este caso posiblemente se combina con subjuntivo o futuro, aunque parece más natural con el primero:

(1b) Posiblemente me matricularé el próximo año.

(1c) Me matricularé posiblemente el próximo año.

Al combinarlo con futuro la aserción informa de una decisión firme y posiblemente se orienta más claramente hacia el complemento de tiempo (1c). En este mismo contexto, si incluimos en lo posible parece menos aceptable.

(1d) ???Me matricularé en lo posible el próximo año

Con ese contenido de «si puedo» son más aceptables las formas si esposible, a ser posible. En el caso siguiente posiblemente se combina con una perífrasis de obligación:

(2a) Posiblemente tengas que evitar la exposición al sol

El hablante no afirma si es verdadera o falsa esa obligación. Su paráfrasis podría ser «que tengas que evitar la exposición al sol es una posibilidad»o «es posible que tengas que evitar la exposición al sol». Posiblemente afecta a todo el enunciado, a toda la acción, incluyendo la modalidad:

Posiblemente [Enunciado: modalidad+ contenido]

Cuando incluimos la forma en lo posible se mantiene la modalidad de obligación, que es deóntica, no epistémica.

(2b) Tienes que evitar en lo posible la exposición al sol.

En lo posible afecta al contenido de la perífrasis tienes que evitar. Aunque se centre fundamentalmente su acción en el verbo predicativo (evitar), afecta en cierto modo, atenuándola, a la modalidad de obligación, rebaja el grado de la imposición.

Modalidad de obligación + oración (verbo+en lo posible).

Como percibimos, hay algunas diferencias entre las dos formas citadas. Posiblemente es un operador de posibilidad epistémico. En lo posible en algunos contextos se comporta como un operador atenuativo de la modalidad deóntica. 
Posiblemente conmuta con quizás. En lo posible con «en lo que pueda(s)»o «si puedes». Establece una limitación a la realización del hecho designado o a la fuerza ilocutiva. En este último caso tiene como efecto una atenuación de los efectos impositivos.

3.1.2. Esta primera hipótesis, surgida de la comparación con un adverbio de posibilidad, viene corroborada por los corpus. En lo posible aparece en distintos contextos. En unos mantiene su contenido léxico originario. El más claro y más cercano a un contenido macroestructural, propio de un operador, es el modal atenuativo.

En ambos contextos su contenido apunta a las posibilidades que tiene el hablante en un contexto concreto. Su paráfrasis sería «en lo que pueda(s), si hay posibilidad». Pero la combinatoria sintagmática con formas verbales y perífrasis provoca efectos discursivos distintos. De ahí los dos empleos de los que hablamos.

a. Valor originario, léxico: «en lo que pueda(s)»

En este contexto en lo posible actúa como complemento de un verbo o un sintagma y aparece integrado, sin formar grupo entonativo independiente. Mantiene su significado base y establece una limitación a la realización de lo dicho: la acción de omitir en (3) se reduce a lo que puede hacer el hablante:

(3) En lo que se refiere a mi trabajo omitiré en lo posible la cronología puesto que se conoce bastante bien la vida de Francia. Me centraré en las diversas facetas de la Dictadura. Basándome en varios testimonios de personas de distintos grupos sociales de la población. La mayoría de ellos fueron testigos de la época. (CORPES, Ferrer Agüero, Luis María: El emperador chino de occidente (El Dr. F.): guión cinematográfico, 2001)

Admite combinatoria con cualquier forma verbal. En el fragmento anterior aparece con un futuro. En (4) lo encontramos con un futuro hipotético y se documenta también con infinitivo (5) o subjuntivo (6), todos ellos de carácter prospectivo. Estamos hablando de algo no realizado, proyectado hacia el futuro. Su alcance también puede variar. En (4) es un sintagma coordinado complemento del verbo reír. Y en lo posible aparece resaltado entre pausas:

(4) Pero me gustaría que lo festejemos, que nos riamos de esto y, en lo posible, de todo lo que está a mano para hacernos reír. Yo también te quiero, guacha. (CORPES, Herrera, Marcos: Ropa de fuego, 2001). 
(5) Ello significa prever situaciones de cambio inherentes a la vida (laborales, familiares, sociales y en general), el mantener en lo posible el control sobre la propia vida, así como procurar activamente el mantenimiento y el mejoramiento de la calidad de la misma, para tener la posibilidad de vivir la vejez en condiciones de bienestar, evitando concepciones prejuiciadas de la vida, de los cambios del envejecimiento y de la senectud. (CORPES, Secretaría de Salud: Programa de Acción: Atención al Envejecimiento, 2001).

(6) Sin embargo, este manejo implica siempre la creación de un estado en cierta medida artificial, que si bien se busca que se asemeje en lo posible a la condición del bosque virgen, es distinto de él. (CORPES, Fundación para la Innovación Agraria: Bosque nativo en Chile: situación actual y perspectivas, 2001).

El hablante no se impone y admite una limitación a esas acciones, según el contexto de realización. Puede ser sustituible por la expresión en lo que pueda(s) o incluso por el otro operador que estudiaremos a continuación: en la medida de lo posible. Si es posible no precisa el concepto de cantidad o medida que expresan estas otras formas.

En otros casos, en lo posible actúa sobre verbos en presente o en pasado. Modifica el contenido verbal: expone un hecho o acción que se dio o se da, no en toda su extensión sino dependiendo de las circunstancias contextuales. Hay, pues, una rebaja, una limitación en la extensión de lo dicho:

(7) Esto ocurrió en 1827, pero fue sólo hacia 1850 en que el abuelo de mi abuela, Don Lorenzo Barrios, mayordomo de la imagen, comienza a restaurar el culto a la virgen y las costumbres de los guanches respecto a ella. La llamó "fiesta de los naturales". El viejo santuario se adecenta y restaura en lo posible. Es en ese momento cuando comienzan a llegar los peregrinos que desde 1863 se hacen masivos. (CORPES, Marrero, Marisol: Las brujas modernas vuelan en la red, 2001).

Puede reformularse como «se adecenta y restaura en lo que puede (pudo) hacerse». En (8) se indica que el deseo era incluirlo todo, pero las circunstancias limitan la extensión hasta ciertos extremos. Se realiza en el grado en el que las circunstancias lo permiten.

(8) Las especies se presentan en orden alfabético, según el orden y/o familia, género y especie. Incluyen, en lo posible, todas las especies exóticas detectadas en el país. Sin embargo, algunos cuadros están incompletos, especialmente 
cuando la cantidad de especies o el bajo grado de conocimiento de las mismas rebasaron el tiempo previsto para el presente estudio. (CORPES, Ojasti, Juhani ... [et al.] [eds.]: Informe sobre las Especies Exóticas en Venezuela. Caracas: Ministerio del Ambiente y de Asuntos Naturales, 2001).

Lo encontramos también con un verbo en pasado, equivalente a «en lo que pudo»:

(9) Durante algunos días, los cuatro o cinco previos a la fecha convenida con el conocido, Yuyo Galves se abstuvo en lo posible de ver vis a vis a sus amigos. (CORPES, Ehrenhaus, Andrés: La seriedad. Barcelona: Mondadori, 2001).

En lo posible en estos contextos es una expresión de limitación, que actúa sobre el contenido verbal ejerciendo un efecto desrealizante (Ducrot, 1995) sobre él. Rebaja el grado de realización del contenido designativo expresado por el verbo. Esta limitación viene de la relación con el contexto y con la capacidad del emisor (o la que le atribuye el emisor a los agentes de las acciones referidas). No expresa modalidad epistémica, sino una limitación a aquello deseable (en futuro o con modalidad prospectiva) o a lo que la realidad permite, aunque no coincida con los deseos del hablante (presente y pasado). A pesar de que su contenido es aún transparente y muy cercano a su significado base, sí es perceptible una fijación en la forma y en su distribución. Actúa como un grupo que se antepone (4) o pospone (9) a su ámbito y en ocasiones aparece entre pausas (8).

El concepto de posibilidad está presente pero expuesto como contenido dictal: «en lo que se puede». Se acerca a «si es posible», pero este contenido procedimental se orienta hacia lo designativo y actúa sobre él. No se entiende como una coordenada que implica la no aserción como verdadero o falso, sino que indica la dependencia de las circunstancias para marcar el grado de realización de un hecho, sea presente, pasado o futuro. Estamos, pues, en un límite muy delgado entre contenido designativo o procedimental, y entre micro y macrosintaxis. Hay cierta fijación en la construcción pero no se cumplen otras condiciones de la gramaticalización. No hablamos, pues, de operador en este empleo.

b. El segundo contexto de uso orienta hacia la modalidad directiva: obligación, instrucción. Se combina frecuentemente con perífrasis como tener que, deber, haber $q u e+$ infinitivos, con infinitivos o segmentos nominales con valor instruccional. En lo posible actúa como atenuativo del acto directivo. Afecta a la fuerza ilocutiva rebajándola. Este contenido relacionado con la capacidad, más que con la posibilidad, introduce la reserva. Esa limitación reduce la fuerza de la imposición, y tiene un efecto como 
mecanismo cortés, aunque no en todos los casos. Se trata de una reducción de la imposición, pero no a través de una estrategia interactiva o una referencia a la voluntad del receptor (si no te importa, por favor) sino por rebajar las posibilidades de realización de un hecho. No se apunta a la libertad del oyente; tiene en cuenta las limitaciones externas que pueden impedirle realizar una acción. Las diferencias en la fuerza ilocutiva del acto directivo dependen de la forma verbal con la que se combina: con imperativos en (10), futuros hipotéticos prospectivos (11) o perífrasis de obligación en (12, 13):

(10) BETINA: En lo posible sea breve, por el tiempo, recuerde que le dije que... (CORPES, Robino, Alejandro: Risas grabadas. www.celcit.org.ar: celcit.org. ar, 2013-04-23).

(11) Se podría organizar para los mismos, programas sencillos, con objetivos concretos de acuerdo a las necesidades e intereses de la comunidad. Las emisiones podrían dirigirse a los artesanos individualmente, o a grupos formados en cada localidad, que podrían reunirse con regularidad para analizar y discutir los mensajes recibidos y en lo posible emprender alguna acción en relación a ellos. En este caso los programas, podrían organizarse con instructores en régimen de tutorías. (CORPES, Miranda de Alvarenga, Alejandra Estelbina: Las artesanías tradicionales del Paraguay. Análisis cualitativo y descripción socioeducativa de sus productores. Asunción: Ministerio de Educación y Cultura, 2001).

(12) Yo no podía poner la mano en el fuego por Fonseca, pero habría jurado ante cualquier tribunal de metodología profesional que era el mejor guía de los posibles para meterse en el berenjenal en el que se estaba metiendo Almudena. Lo que había que hacer era poner siempre entre paréntesis las informaciones que diera, contrastarlas en lo posible y no jugársela. (CORPES, M. Reverte, Jorge: Gálvez en la frontera. Madrid: Santillana, 2001).

(13) La sobreexcitación nerviosa es perjudicial a la digestión y debe evitarse en lo posible. Los adelgazamientos rápidos, tan en boga hoy en día, son peligrosos, ya que exponen al estallido de una tuberculosis o de un trastorno digestivo (CORPES, Goldemberg, I.: El nombre del padre. Lima: Alfaguara, 2001).

En estos contextos en lo posible hace pasar de la obligación a la recomendación. Otras posibilidades constructivas son perífrasis como tratar de+ verbo, intentar + verbo, o contenidos verbales que tienen ese efecto directivo o proyectivo: se recomienda, se pretende; estructuras que expresan recomendaciones atenuadas: es preferible, es mejor; o sustantivos que también actúan como formas directivas indirectas: el objetivo (meta, idea, propósito...) es. Veamos algunos ejemplos a continuación: 
(14) Es necesario: 1) purificar las listas de especies repetidas; 2) completar, en lo posible, el inventario con los grupos aún no incluidos, tales como arácnidos, anélidos, nemátodos, hongos y otros agentes patógenos, así como varios taxa del ambiente marino; 3) consultar los productos del trabajo con especialistas nacionales para agregar especies omitidas y verificar la asignación de las especies por categorías de invasoras, establecidas (CORPES, Ojasti, J. ... [et al.] [eds.]: Informe sobre las Especies Exóticas en Venezuela, 2001).

(15) Domínguez: Totalmente cuestarriba, padre. Lo mejor será conservar en lo posible las estructuras de gobierno interno. No tiene caso alborotar a la indiada. (CORPES, Tovar, Juan: Horas de gracia. Último sueño de Agustín primero. Onirofarsa. México D. F.: Jus, Compañía Nacional de Teatro, 2010).

(16) La mayoría de los vecinos coinciden en señalar que es necesario incrementar la presencia policial con el objetivo de intentar paliar en lo posibl$e$ este problema que vienen padeciendo, según aseguran, desde hace años. No obstante todos ellos afirman que la solución radica en concienciar a la juventud para que aprendan a divertirse, sin tener que recurrir a la violencia (CORPES, Faro de Vigo, 2001)

En ocasiones se combina con futuro, cuando se presenta como una planificación de hechos o instrucciones, o con infinitivos:

(17) Esta actividad se realizará de manera preliminar en gabinete y luego se profundizará el análisis en talleres municipales y/o en lo posible en un taller departamental, donde participarán los actores sociales e institucionales. En esta fase se procederá a revisar la propuesta preliminar del equipo técnico y a complementarla de acuerdo a las sugerencias y aportes de los actores. (CORPES, Ministerio de Desarrollo Sostenible y Planificación ... [et al.]: Guía metodológica para la formulación de los planes departamentales de ordenamiento territorial, 2001).

Aparte de estos casos de modalidad exhortativa, los más frecuentes, hemos encontrado alguna ocurrencia con actos valorativos o desiderativos como el siguiente:

(18) Toda literatura debería ser terapéutica?

- No estoy para establecer leyes. Mi literatura lo es y en lo posible ojalá lo hubiera. Pero los seres también necesitan divertirse, bailar y entretenerse, por ello también estoy por la literatura de diversión. (CREA, Excélsior, 05/09/2000) Encontramos un caso en que complementa al verbo gustar, pero parece ser 
una atracción a la posición adelantada de un complemento del verbo pensar, objeto de me gusta.

(19) Me gusta en lo posible pensar y proyectar cada acto de mi existencia. Intento no dejar nada al azar, algunas veces vanamente, lo confieso. (CREA, L. Llongueras: Llongueras tal cual. Anécdotas y recuerdos de una vida, 2001)

En cuanto al alcance de en lo posible, puede ser un sintagma (20), toda la oración o todo el predicado. En (21) afecta a todo el enunciado («de pelo de marta »):

(20)Escoger leche y derivados bajos en grasa, en lo posible sin grasa.(CORPES,"Cómo seleccionar sus alimentos». Revista de Nutrición XXI. Santiago de Chile: Instituto de Nutrición y Tecnología de los Alimentos de la Universidad de Chile, 2002).

(21) -Vamos, trae tinta china y un pincel. En lo posible, de pelo de marta. (CORPES, Maronna, Jorge; Pescetti, Luis María: Copyright: plagios literarios y poder político al desnudo. Barcelona: Plaza \& Janés, 2001).

3.1.3. La expresión considerada tiene movilidad: aparece también en posición inicial, con pausas o sin ella. En el primer caso focaliza la información y actúa como complemento periférico marco. Establece una primera condición que limita la imposición que sigue. Por una parte, esta limitación es cortés, porque ofrece opciones al receptor, pero también destaca esta información, orienta la interpretación del receptor hacia el acto de habla y lo condiciona a las posibilidades de realización en el contexto, no a la voluntad del individuo. Su contenido es semejante al de si (te) es posible. En esta posición ya lo encontramos en el CORDE, al inicio del siglo XX:

(22) En lo posible han de ser entresacadas de las "Lecturas B" y las "Lecturas C”. Su fin principal es el estudio de la Gramática científica. Han de ser breves trozos graduales; arreglados de tal suerte, que una lección bien aprendida, sirva de medio para emprender los estudios sucesivos. (CORDE, J.I. Lezcano: Programa analítico de castellano del prof. Juan Inocencio Lezcano, 1931).

En CREA y CORPES son menos frecuentes en posición inicial que en posición intercalada, como señalaremos en el punto 4, pero hay bastantes casos. 
(23) En lo posible trate de no nadar y de no hacer ejercicios bruscos para "calentarse", ya que produciría el efecto contrario. (CREA, J.N. Artigas: Manual de sobrevivencia, 1991).

Del primer valor, limitativo, podemos citar (24) aunque mantiene cierto valor de recomendación:

(24) Para la prueba se empleó $20 \mathrm{mg}$ de extracto total de la planta. En lo posible el compuesto debe ser soluble en frío o en baño maría en dimetilsulfóxido (DMSO) que es el solvente universal e inocuo para la $A$. salina. (CORPES, Abdo, Susana: «Capítulo II. Fitoquímica. La defensa química de las plantas superiores», 2003).

En CORPES encontramos fundamentalmente su uso con actos directivos: consejos, recomendaciones, obligaciones... El siguiente pertenece a una descripción en presente, aunque proyectada al futuro, por el contenido de planificación, deseo y no realización de trato de:

(25) Nunca la pensé mucho. Todo fue llegando de a poco y, con el tiempo, se fue armando de la manera que está hoy. En lo posible trato de poner cosas que tengan un significado que vaya más allá de lo puramente estético, pero claro, eso no se puede dar con todo. Además, es el tiempo y lo que uno vive lo que también hace que los momentos se identifiquen con los objetos y así éstos adquieran una importancia especial. (CORPES, Maldonado, Ari: «Puertas adentro». ED. Santiago de Chile: ed.cl, 2012-06).

Como operador atenuativo, lo encontramos con diferentes formas verbales, como ya explicamos:

(26) Prevención

- Limpiar la vivienda y cambiar la ubicación de los objetos pesados (...)

- En lo posible, eliminar los reservorios del parásito que ocasiona el mal: perros, gatos, cerdos y murciélagos. (CREA, El Salvador Hoy, 06/04/1997)

(27) A todos nos vendría bien una cura de minúsculas para rebajar humos retóricos. En lo posible nos tendríamos que atener a la norma estricta de que sólo van con mayúscula los nombres propios de personas o de entes personalizados. Las excepciones, con permiso por escrito. (CREA, A. de Miguel: La perversión del lenguaje, 1994) 
(28) En lo posible, la capacitación especial para un trabajo u ocupación debe comenzar después del ciclo de cultura general de la educación secundaria, o a la par de él, pero esto no impide que por razones económicas y sociales se ponga al final de la escuela primaria y aun antes. (CREA, L.A.Lemus: Administracion, dirección y supervisión de escuelas, 1975)

(29) Consejos seguros. Al salir a pasear - En lo posible utilice la tarjeta de crédito o cheques de viajero. No muestre grandes cantidades de dinero, y una buena idea puede ser separar el cambio chico para compras pequeñas, de los billetes gordos. - Cuando utilice la tarjeta de crédito, verifique que le hayan devuelto su tarjeta y no cualquier otra. - No lleve su billetera en el bolsillo trasero del pantalón. (CREA, H. de Dios: Miami, 1990)

3.1.4. Como podemos ver, se acerca al operador discursivo por su distribución, fijación formal y contenido procedimental (función discursiva de atenuación). La limitación es el contenido base, que se desarrolla en el plano dictal, como complemento verbal, o en el modal, afectando a la fuerza ilocutiva. En ambos se presenta una opción al oyente y a veces se percibe cierto contenido cercano a lo deseable. Así sucede en el uso como complemento verbal.

(30) El esperaba que fuera sólo eso, un paréntesis, en lo posible no muy largo, el tiempo suficiente para que ella se diera cuenta que era él quien podía darle lo que ella necesitaba. (CORPES, Arcos Levi, René: Después de todo. Santiago de Chile: Planeta, 2001)

Esta limitación actúa como una opción que implica que la modalidad exhortativa no se realice con toda su fuerza. Es una limitación que adquiere una función atenuativa, como una opción al mandato. Las funciones discursivas de en lo posible son, pues:

a) Limitación del contenido dictal — con presente y pasado. No tiene contenido cortés o descortés y actúa como complemento del verbo. Liga el hecho realizado a las posibilidades contextuales de realización. Puede ser sustituible por en lo que pueda(s) o si es posible. El primero establece cierto contenido cualitativo que se pierde en el segmento en lo posible.

b) Atenuación de la fuerza ilocutiva: generalmente de la modalidad exhortativa. Aparece entre pausas. Se combina con futuro, con perífrasis de obligación o proyectivas. Su contenido es rebajar la fuerza modal y pasar de la obligación o imposición a la recomendación. El hablante acepta que el oyente no tenga opciones o que el contexto no le permita realizar lo propuesto. En este sentido es cortés. En este empleo es un operador, ya que ha desarrollado un contenido procedimental. 
El primero se mueve en el campo del contenido dictal del verbo. En el segundo caso actúa en el plano modal, pero como atenuador de modalidades directivas.

En cuanto a la pregunta sobre su grado de fijación como operador, hay que decir que no suele aparecer entre pausas; solo en algunos casos aparece focalizado, formando grupo entonativo independiente, aunque no parece haber una sistematicidad en ello. Sí tiene movilidad en el enunciado y ocupa diversas posiciones. En el empleo a) es un limitador argumentativo que se aplica sobre el contenido verbal, reduce la acción según sus circunstancias contextuales. Solo lo consideramos operador en el segundo empleo (b), ya que desarrolla un contenido procedimental, atenuativo, que actúa macroestructuralmente sobre la modalidad y tiene efectos argumentativos. Sería, como hemos venido diciendo, operador atenuativo de la modalidad exhortativa, de actos directivos. Reduce los efectos y por ello puede usarse como estrategia atenuativa.

En este como en el siguiente elemento se alude a un enfrentamiento entre lo que es posible (porque lo permiten las circunstancias contextuales) y lo que es deseado por el hablante e implica al oyente. Este enfrentamiento con estas formas se presenta siempre de forma atenuada, con la conciencia colaborativa del hablante de que las circunstancias pueden impedir la realización de lo proyectado.

En cuanto a su aparición en los corpus, CORDE lo documenta desde el siglo $\mathrm{XVI}$, aunque en sus inicios el valor desarrollado es el primero. Encontramos los primeros datos del atenuativo a partir del siglo XVIII: la tabla 1 muestra la evolución y la tablas 2 y 3 recogen los usos en CREA (limitada la búsqueda a los últimos años: 2000-2004) y CORPES (2013-2016)9.

\begin{tabular}{|l|c|c|c|c|c|c|}
\hline & XVI & XVII & XVIII & XIX & XX & TOTAL \\
\hline En lo posible & $5(5 / 0)^{10}$ & $12(12 / 0)$ & $38(20-18)$ & $429(181 / 248)$ & $401(173 / 228)$ & 885 \\
\hline
\end{tabular}

Tabla 1. En lo posible en CORDE

\begin{tabular}{|c|c|c|}
\hline Corpus & $\begin{array}{c}\text { CREA: posición inicial/intercalada } \\
(\mathbf{2 0 0 0 - 2 0 0 4})\end{array}$ & $\begin{array}{c}\text { CORPES: posición inicial/intercalada } \\
(\mathbf{2 0 1 3 - 2 0 1 6 )}\end{array}$ \\
\hline En lo posible & $140\left(2 / 138^{11}\right)$ & $143(13 / 130)$ \\
\hline
\end{tabular}

Tabla 2. En lo posible en CREA y CORPES: posición

${ }^{9}$ El CORPES recoge los datos de CREA en los años 2000-2004. Por el volumen de datos y porque nuestro objetivo era conocer la situación actual, hemos incorporado este otro corpus. De ahí que lo hayamos limitado a los 4 últimos años del mismo.

${ }^{10}$ Aparece el número total y entre paréntesis los valores $1 / 2$.

${ }^{11}$ Aunque en la página de CREA aparecen 141 casos en posición intercalada, hay tres casos repetidos, que no hemos computado. 


\begin{tabular}{|l|c|c|}
\hline \multicolumn{1}{|c|}{ Corpus } & $\begin{array}{c}\text { CREA: limitación/operador ate- } \\
\text { nuativo (2000-2004) }\end{array}$ & $\begin{array}{c}\text { CORPES: limitación/operador } \\
\text { atenuativo (2013-2016) }\end{array}$ \\
\hline En lo posible & $140(55 / 85)$ & $143(37 / 106)$ \\
\hline
\end{tabular}

Tabla 3. En lo posible en CREA y CORPES: distribución de los dos usos

\subsection{En la medida de lo posible}

3.2.1. En esta segunda expresión el concepto de posibilidad aparece en la extensión del sintagma. Esta construcción no está recogida en el DRAE como un elemento con valor único. No está reconocido como operador en los repertorios lexicográficos de conectores: el diccionario en línea de Briz-Pons-Portolés (2008-actualidad), Fuentes Rodríguez (2018[2009]) o Santos Río (2003). Este último solo recoge a ser posible como adverbio condicional.

Este sintagma nominal incluye un sustantivo nuclear, medida, que apunta a lo cuantitativo. Semánticamente, posible se dirige hacia lo modal. La unión de ambos contenidos determina la dirección en la que va a operar la construcción, con comportamientos diversos.

Esta construcción está fijada, ya que no admite variación morfemática de número: *en las medidas de lo posible, *en la medida de los posibles, aunque sí hay alguna variante: en la medida de mis/tus/sus posibilidades, pero esta es otra expresión que estudiaremos a continuación. Tampoco admite cambio de determinante: *en una medida de lo posible. Sin embargo, en el corpus encontramos algunos testimonios que indican que estamos ante una construcción sintácticamente libre que puede coordinarse con otro complemento: «en la medida de lo posible $+y /$ pero también+$\mathrm{de}+\mathrm{sn} \gg$, como documentan los fragmentos siguientes.

(31) Si logramos salvar, vigorizar y dar impulso a este grupo de ciudades, podremos resistir mucho mejor la hecatombe urbanística que se nos avecina, podremos también salvar en la medida de lo posible y lo compatible con el progreso y el desarrollo económico la estructura orgánica del pasado y buena parte de un depósito cultural que debemos a todo trance preservar si tenemos espíritu clarividente, pues a la vez que el mundo avanza a galope tendido hacia la uniformidad e igualitarismo de las sociedades industriales, también se despierta, conforme aumenta la disponibilidad de ocio en las masas de una afluent society, el deseo de encontrar mundos diferentes e insospechados. (CORDE, Chueca Goitia, Fernando: Breve historia del urbanismo, 1968).

(32) Vigilé de cerca ese proceso y no me separé de Iglesias sino lo indispensable: era mi oportunidad más segura de filtrarme en el mundo prohibido y 
no lo iba a malograr por errores groseros. Traté así de permanecer a su lado en la medida de lo posible, pero también de lo insospechable. Lo cuidaba, le leía algún libro de Kropotkin, le conversaba sobre el Apoyo Mutuo, pero sobre todo, observaba y esperaba. (CORDE, Sábato, Ernesto: Sobre héroes y tumbas, 1961).

Aún hay algún caso en que la construcción se coordina con otros complementos directamente relacionados con el verbo nuclear, lo que refuerza la hipótesis del funcionamiento oracional, microsintáctico. Así, en (33) se muestra como complemento circunstancial unida a otro, «en nombre de la patria »:

(33) Y ahora juzgad de la protesta de los imbéciles ante nuestra idea de pedir, en nombre de la patria y en la medida de lo posible, profesores extranjeros. (CORDE, Tamayo, Franz: Creación de la pedagogía nacional. 1910).

La construcción tiene movilidad en el enunciado, puede ocupar diferentes posiciones: inicial o intercalada fundamentalmente. Más rara es su aparición en posición final, aunque también encontramos casos.

En posición inicial establece un marco, como muchos de los elementos con contenido procedimental, que por ello adoptan una función en el margen izquierdo: establece las condiciones de realización de lo que va a seguir. A partir de ahí desarrolla otras funciones. Si estas se mueven en el marco procedimental podremos hablar de un cambio semántico y un paso a lo discursivo: establecer un contenido indexical, que apunta a aspectos relacionados con el hablante, la argumentación y la modalidad.

3.2.2. En principio, podemos afirmar que en la medida de lo posible desarrolla el mismo contenido base de en lo posible: establece una limitación a la acción anterior. Este contenido de limitación se desarrolla en dos contextos:

a) Limitación del contenido verbal: generalmente en un contexto verbal de presente o pasado. Se aplica a la acción verbal del enunciado pero también podría tener como alcance un segmento.

(34) Desde muy niño, obsesionado por mi inferioridad congénita, me apliqué a disfrazarla en la medida de lo posible, ensayando ante el espejo las posturas y ángulos más propicios. (CORDE, Mujica Láinez, Manuel: Bomarzo, 1962).

En este caso afecta al infinitivo, no al grupo verbal completo. No es «me apliqué en la medida de lo posible», sino «disfrazarla todo lo que podía». Ambos con- 
tenidos, posible y medida, siguen manteniéndose con su valor originario. Por tanto, estamos ante un complemento del verbo, aunque, como iremos viendo, su contenido se va fosilizando y adopta un valor procedimental.

(35) Les había explicado a mi manera la ópera de Wagner. Charles me pidió que le ofreciese una muestra de la música, si podía recordarla, y entonces yo, al piano, reproduje en la medida de lo posible algunos temas: los que cantaba Tristán y los que cantaba Isolda. (CORDE, Torrente Ballester, Gonzalo: Don Juan, 1963).

(36) Pero lo que es imposible hacer dignamente, es santo y piadoso hacerlo en la medida de lo posible. (CORDE, Maldonado, Luis: La plegaria eucarística. Estudio de teología bíblica y litúrgica sobre la misa, 1967).

Se confronta «hacer dignamente» y «hacer en la medida de lo posible». La construcción resulta en este contexto difícil de entender.

b) Atenuación de la modalidad exhortativa o de obligación.

Como vemos, la composición del elemento, medida (cuantitativo) yposible (modalidad), se ve reflejada icónicamente en sus empleos discursivos. En este contexto es una construcción camino de fijarse como operador argumentativo de limitación:

(37) La consideramos fundamental ya que a través de ella y sus asociaciones, se trató de dinamizar un pasado que puede permanecer estático si se basa en los análisis simplemente monumentales o altamente descriptivos, pero que también puede dinamizarse a partir de un estudio de carácter global, en la medida de lo posible. (CORDE, C. Huapaya: Informe preliminar sobre el Proyecto de Arqueología y Computación del material del Complejo Pando, 1974)

Podríamos sustituirla por si es posible (dinamizarse). Repite el valor de posibilidad ya expuesto en puede. Pero hay una diferencia: en la medida de lo posible establece una referencia a lo cuantitativo: «en la extensión que podría afirmarse». En este caso la posición de comentario al final también es propia de un marcador, con el valor de reinterpretación: volver a lo dicho y añadir una limitación, lo que hace reducir la fuerza a la aserción.

Es más claro como operador cuando actúa como atenuativo de la modalidad. De nuevo encontramos la expresión combinada con perífrasis de obligación o con instrucciones, todas con contenido prospectivo. Ofrece opciones al oyente, con lo que aporta un contenido cortés: 
(38) El léxico archivo debe, en la medida de lo posible, registrar todo vocablo local. (CORDE, Casares, Julio: Introducción a la Lexicografía moderna, 1950).

En este caso se intercala en medio de la perífrasis.

$\mathrm{Al}$ aplicar una limitación atenúa el valor ilocutivo de las peticiones, órdenes, y las convierte en recomendaciones: aquí ya tenemos un proceso de abstracción más grande, se pierde el concepto de medida. En su distribución, aparece a veces antepuesto y utiliza las pausas. Se acerca en su valor a en loposible:

(39) Luego es preciso, en la medida de lo posible, evitar las repeticiones, que se hacen frecuentemente de una manera maquinal, pero concentrar toda la fuerza de la atención sobre el hecho o la idea que se quieren retener. (CORDE, González Llana, Félix: Traducción de Ideas modernas acerca de los niños, de Alfred Binet, 1913).

(40) El código, en la medida de lo posible, debe indicar las características del objeto codificado. (CORDE, Sancho Rodríguez, J.: La información y su representación [Informática básica], 1974).

Este valor no anda tan lejos del prospectivo, ya que en realidad aquí no se afir$\mathrm{ma}$, sino que se plantean recomendaciones sobre acciones futuras. Véase el siguiente caso, en el que el contenido prospectivo está en el semantismo del verbo remediar y en la construcción final (para remediar), que implica una actuación con el propósito de modificar una situación:

(41) La alfabetización y la educación de adultos se han apoyado en la radio y la televisión para remediar el problema en la medida de lo posible. (CORDE, García Jiménez, Jesús: Teoría de los contenidos de la Televisión, 1965).

La diferencia con los usos modales es que aparece sin pausas y su incidencia inmediata es el verbo remediar.

Podemos hablar, pues, de un operador discursivo por su grado de fijación formal, por su fijación funcional (la extraproposicionalidad) y semántica: el contenido procedimental que desarrolla. En este último aspecto tenemos que hacer algunas aclaraciones. En la medida de lo posible aparece entre pausas en cualquiera de sus funciones, como hemos ido viendo, aunque sea más frecuente en el uso modal. En ambos puede aparecer así cuando se focaliza la información.

En un contexto de futuro, en la medida de lo posible refuerza el contenido de planificación (instrucciones), que admite cambios: 
(42) Especificaba el reglamento que si el número de inscritos era inferior a dieciséis, y éste era el caso, se establecerían cuatro grupos, formados, "en la medida de lo posible, por equipos del mismo valor y de méritos iguales". (CORDE, Mercé Varela, Andrés: Traducción de Fútbol. La Copa del Mundo de Jules Rimet, 1955).

Es el desiderátum, lo que se pretende, aunque sin que pueda asertarse. El operador reduce la fuerza asertiva y lo lleva al plano de lo prospectivo.

En el caso siguiente lo tenemos con tratar de, con una proyección hacia el futuro. En estos casos podría ser sustituible por si es posible o en la medida de sus posibilidades:

(43) Al final, intentaremos ampliar el panorama, en la medida de lo posible, hasta sus aspectos sociales, como base del planteamiento anterior. (CORDE, J.A. Maravall: Las comunidades de Castilla, 1963-1979).

Sin embargo, no serían totalmente equivalentes. Con si es posible el hablante abre una posibilidad inferencial a la no realización. En la medida de lo posible y en lo posible parecen usarse en contextos en los que el hablante presupone que se realizará el hecho aunque se duda del grado de realización. Por ejemplo, en el fragmento siguiente (44) la manifestación se afirma, aunque no el grado. Si ponemos si es posible, la voluntad de manifestar puede no llevarse a efecto:

(44) Recuérdese la definición de Josiah Royce: "La fidelidad es la voluntad de manifestar, en la medida de lo posible, lo eterno; es decir, la consciente y suprahumana unidad de vida en la forma de los actos de un yo individual." (CORDE, Rosales, Luis: Cervantes y la libertad, I, 1960).

Cuando va con perífrasis proyectivas (intentar conseguir X), en la medida de lo posible afecta no al verbo auxiliar, sino al contenido del verbo predicativo.

(45) Entonces pasa a ser responsabilidad del director el descubrir qué es lo que la gente quiere saber y facilitarles las respuestas y la información en la medida de lo posible. (Ob. cit., págs. 163 y 164). (CORDE, Carrasco Belinchón, Julián: Manual de organización y métodos. I. Funciones directivas, 1966).

Las circunstancias sintagmáticas del operador están muy determinadas. Podríamos hablar de una construcción formada por «verbo con contenido modal 
atenuativo o prospectivo, o un entorno de futuro (determinación por perífrasis o por formas verbales)». Aquí encontramos tratar de, deber de, intentar+ infinitivo, futuro y futuro hipotético. Asimismo, el valor prospectivo básico en esta unidad se encuentra en el contenido léxico de las palabras que constituyen el alcance o que preceden a este alcance: intentar, evitar, procurar, pretender, sustantivos como intento, recomendación, idea, propuesta... o construcciones sintácticas como las estructuras finales con para.

(46) En la medida de lo posible, les dejaremos hacer, si no ponen en peligro su seguridad. (CREA, Penella, Manuel: Tu hijo: genio en potencia. Las claves fundamentales para su educación, 1995).

(47) En la medida de lo posible deberemos procurar que nuestra vivienda disponga de toda la luz natural que sea posible, pues, como dice el refrán: "Donde entra el sol no entra el médico”. (CREA, Bueno, Mariano: El libro práctico de la casa sana, 2004).

3.2.3. En el corpus CORDE, aparecen testimonios de en la medida de lo posible en 42 ocasiones, a partir de 1800. En el siglo XIX documentamos 7 casos y 35 en el XX. El reparto por valores aparece en la tabla 4 . La tabla 5 recoge los resultados de CREA y CORPES según su posición y valor:

\begin{tabular}{|l|c|c|}
\hline \multicolumn{1}{|c|}{ CORDE: 42 (A PARTIR 1800) } & Inicial & Intercalado \\
\hline Limitación & 1 & 13 \\
\hline Modal & 6 & 22 \\
\hline
\end{tabular}

Tabla 4. En la medida de lo posible en CORDE

\begin{tabular}{|c|c|c|c|c|}
\hline Corpus & $\begin{array}{c}\text { CREA (2000-2004): } \\
\text { inicial/intercalado }\end{array}$ & $\begin{array}{c}\text { CORPES } \\
(2013-2016): \\
\text { inicial/intercalado }\end{array}$ & $\begin{array}{c}\text { CREA } \\
(2000-2004): \\
\text { limitación/operador } \\
\text { atenuativo }\end{array}$ & $\begin{array}{c}\text { CORPES } \\
\text { (2013-2016): } \\
\text { limitación/operador } \\
\text { atenuativo }\end{array}$ \\
\hline $\begin{array}{l}\text { En la medida } \\
\text { de lo posible }\end{array}$ & $8 / 108$ & $11 / 100$ & $36 / 80$ & $49 / 62$ \\
\hline
\end{tabular}

Tabla 5. En la medida de lo posible en CREA y CORPES 


\subsection{En la medida de mis/tus/sus posibilidades}

En la medida de sus/mis/tus posibilidades es un sintagma nominal complejo que aparece como complemento circunstancial del verbo. Mantiene variabilidad en concordancia con el referente de la acción (generalmente el hablante (48) o incluso el oyente (49)).

(48) En razón de ello, las personas que me informaron pudieron cometer un ligero error con la sobreposición del nombre de José Luis Navas, error que, en la medida de mis posibilidades, estoy absolutamente dispuesta a rectificar. (CREA, El País, 10/07/1980)

(49) Parecía sincero cuando afirmó desconocer en qué prisión se hallaba. "Es como si se lo hubiese tragado la tierra", dijo. Le acorralaste en la medida de tus posibilidades, si bien en aquel combate entre dos espíritus quebradizos estabas perdiendo terreno por momentos. (CREA, J.A.Gabriel y Galán: El bobo ilustrado, 1986)

En el caso de en la medida de sus posibilidades encontramos su primer empleo en CORDE a partir del siglo XX. No hay casos anteriores. Y en este siglo encontra$\operatorname{mos} 7$ casos intercalados ${ }^{12}$. En estos fundamentalmente hay una orientación hacia el futuro (en un complemento final), en consonancia con el semantismo del sustantivo posibilidades:

(50) De ahí la presión desesperada que ejerce Hitler sobre Polonia para romper, en la medida de sus posibilidades, el cerco que se va formando en torno a la amenaza nazi. (CORDE, Anónimo, Nota internacional. Lo que va de ayer a hoy, 1934).

Mantiene el valor léxico y sería equivalente al contexto 1 de en la medida de lo posi$b l e^{13}$. Es una construcción aún no fijada ya que admite variación formal: aparece en plural (nuestras, pero no vuestras posibilidades), según muestra (51), obtenido del CREA.

\footnotetext{
${ }^{12}$ De los 7 casos, 6 son prospectivos. No hay ningún caso en posición inicial.

${ }^{13}$ Podría pensarse, como ha sugerido un evaluador, que la construcción libre, en la medida de sus posibilidades, apunta a una persona concreta (Hitler, en este caso). Puede depender de los aspectos ligados a él y a su realidad concreta. Si lo sustituimos por en la medida de lo posible, el contenido es más general, más abstracto. Este es uno de los rasgos diferenciadores del operador discursivo fijado, que elabora un contenido procedimental constante, aplicable a todos los contextos (vid. Fuentes Rodríguez, 2019).
} 
(51) -Si tienes hambre o sed, dínoslo y procuraremos satisfacerte en la medida de nuestras posibilidades. Y si luego quieres, elige a la que más te guste de nosotras y sacia tu fogosidad. (CREA, E.Mendoza: La verdad sobre el caso Savolta, 1975)

Encontramos pocas ocurrencias de en la medida de mis/tus/nuestras posibilidades según muestra la tabla 6. En el caso de en la medida de sus posibilidades encontramos más ejemplos. En CORPES, por su parte, acumula esta expresión 931 ocurrencias, como muestra la tabla, y aparecen algunas ocurrencias en CORDE, como la siguiente:

(52) Luego, policías distribuidos convenientemente en las tribunas, impidieron, en la medida de sus posibilidades, aquel desencadenamiento de escándalo, alboroto y fetidez. (CORDE, Mercé Varela, Andrés: Traducción de Fútbol. La Copa del Mundo de Jules Rimet. Barcelona: Juventud, 1955).

\begin{tabular}{|l|c|c|c|c|c|}
\hline & $\begin{array}{c}\text { En la medida de mis } \\
\text { posibilidades }\end{array}$ & tus & sus & nuestras & vuestras \\
\hline CORDE & 0 & 0 & 7 & 3 & 0 \\
\hline CREA & 11 & 2 & 61 & 22 & 0 \\
\hline CORPES & 22 & 5 & 931 & 29 & 0 \\
\hline
\end{tabular}

Tabla 6. En la medida de mis posibilidades y variantes en los corpus analizados

Podría parecer, tras la lectura de esta tabla, que predomina la referencia al hablante (mis, nuestras posibilidades) pero, aunque tus presente pocas ocurrencias y vuestras ninguna, en sus posibilidades se incluye la referencia a una tercera persona y a ustedes, plural formal referido al oyente.

\section{EN LO POSIBLE, EN LA MEDIDA DE LO POSIBLE: COMPARATIVA DE SU PRESENCIA EN LOS CORPUS}

Los resultados parciales de ambas formas nos permiten establecer alguna comparación en cuanto a su grado de presencia, el uso predominante o su fijación en el sistema. La tabla 7 muestra el predominio de en lo posible sobre en la medida de lo posible, incluso en etapas anteriores. En todos los corpus, la función intercalada es la preferida. 


\begin{tabular}{|l|c|c|c|}
\hline \multicolumn{1}{|c|}{ Corpus } & $\begin{array}{c}\text { CREA (2000-2004): } \\
\text { Inicial/intercalado }\end{array}$ & $\begin{array}{c}\text { CORPES (2013-2016): } \\
\text { Inicial/ intercalado }\end{array}$ & $\begin{array}{c}\text { CORDE: } \\
\text { Inicial /intercalado }\end{array}$ \\
\hline En lo posible & $2 / 138^{14}$ & $13 / 130$ & $6 / 879$ \\
\hline $\begin{array}{l}\text { En la medida de lo } \\
\text { posible }\end{array}$ & $8 / 108^{15}$ & $11 / 100$ & $7 / 35$ \\
\hline
\end{tabular}

Tabla 7. Presencia de las dos expresiones en los corpus

Los resultados de ambos en estos siglos iniciales son diferentes. En lo posible se documenta antes:

\begin{tabular}{|l|c|c|c|c|c|c|}
\hline & XVI & XVII & XVIII & XIX & XX & TOTAL \\
\hline En lo posible & $5(5 / 0)^{16}$ & $12(12 / 0)$ & $38(20-18)$ & $429(181 / 248)$ & $401(173 / 228)$ & 885 \\
\hline $\begin{array}{l}\text { En la } \\
\text { medida de lo } \\
\text { posible }\end{array}$ & 0 & 0 & 0 & $7(0 / 7)$ & $35(7 / 28)$ & 42 \\
\hline
\end{tabular}

Tabla 8. Presencia en CORDE

La tabla 9 (y la figura siguiente) recoge la presencia en CREA y CORPES atendiendo a la posición y a la frecuencia de los dos valores. La conclusión es clara: preferencia por la posición intercalada y preferencia por la función como atenuativo, lo que nos hace confirmar la convivencia de las dos funciones en el momento actual y el desarrollo de un operador ya fijado.

\begin{tabular}{|l|c|c|c|c|}
\hline Corpus & $\begin{array}{c}\text { CREA (2000-2004): } \\
\text { inicial/intercalado }\end{array}$ & $\begin{array}{c}\text { CORPES } \\
(\mathbf{2 0 1 3 - 2 0 1 6 ) :} \\
\text { inicial/intercalado }\end{array}$ & $\begin{array}{c}\text { CREA } \\
(\mathbf{2 0 0 0 - 2 0 0 4 ) :} \\
\text { limitación/operador } \\
\text { atenuativo }\end{array}$ & $\begin{array}{c}\text { CORPES } \\
\text { (2013-2016): } \\
\text { limitación/operador } \\
\text { atenuativo }\end{array}$ \\
\hline En lo posible & $2 / 138$ & $13 / 130$ & $55 / 85$ & $37 / 106$ \\
\hline $\begin{array}{l}\text { En la } \\
\text { medidade } \\
\text { loposible }\end{array}$ & $8 / 108$ & $11 / 100$ & $36 / 80$ & $49 / 62$ \\
\hline
\end{tabular}

Tabla 9. Distribución por posición y funciones

\footnotetext{
${ }^{14}$ En todo el corpus CREA su presencia es 37/802.

${ }^{15}$ En todo el corpus CREA los datos son 22/357.

${ }^{16}$ Valor $1 / 2$.
} 


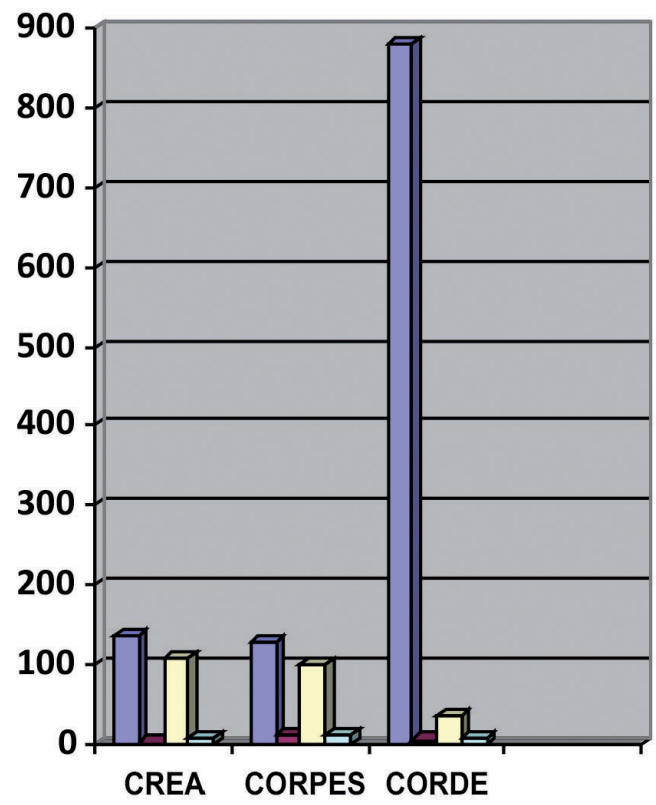

\begin{tabular}{|c|}
\hline en lo posible \\
intercalado \\
$\square$ en lo posible inicial \\
$\square$ en la medida de lo \\
posible intercalado \\
$\square$ en la medida de lo \\
posible inicial
\end{tabular}

Esta conclusión se ve apoyada por los datos. Como en CORPES hemos seleccionado un fragmento más pequeño de tiempo (de 2013 a 2016 inclusive), podemos confrontarlo con los últimos años del CREA: de 2000 a 2004. Podríamos establecer la siguiente secuencia: CREA 1975-1999, CREA 2000-2004 y CORPES 20132016, que nos permitirá ver el progreso en el tiempo del desarrollo del operador. Los datos se muestran en la tabla 10.

\begin{tabular}{|l|c|c|c|}
\hline \multicolumn{1}{|c|}{ Corpus } & $\begin{array}{c}\text { CREA 1975-1999: } \\
\text { número total } \\
\text { (inicial/intercalado) }\end{array}$ & $\begin{array}{c}\text { CREA 2000-2004: } \\
\text { número total } \\
\text { (inicial/ intercalado) }\end{array}$ & $\begin{array}{c}\text { CORPES (2013-2016): } \\
\text { número total } \\
\text { (inicial /intercalado) }\end{array}$ \\
\hline En lo posible & $699(35 / 664)$ & $140(2 / 138)$ & $143(13 / 130)$ \\
\hline $\begin{array}{l}\text { En la medida } \\
\text { de lo posible }\end{array}$ & $263(14 / 249)$ & $116(8 / 108)$ & $111(11 / 100)$ \\
\hline
\end{tabular}

Tabla 10. Progresión de los resultados en CREA y CORPES

En CREA se ve más claramente el incremento. CORPES presenta menor presencia, aunque puede deberse a la limitación del corpus en estos últimos años. 
En el corpus MEsA no encontramos apenas ejemplos. No hay casos en los siguientes corpus: Facebook (264 305 palabras), Twitter (299 122 palabras), WhatsApp (496 157 palabras), YouTube (382 637 palabras). En otros sí tenemos algún caso: blogs (400 772 palabras), foros (367 852 palabras), Instagram (332 575 palabras), páginas web (460 265 palabras), pero son poco significativos. Esto viene a refrendar la hipótesis inicial de la que partimos sobre su uso preferente en lo escrito y en lenguaje formal.

\begin{tabular}{|l|c|c|c|c|c|c|c|c|}
\hline & Blogs & Foros & Instagram & $\begin{array}{c}\text { Páginas } \\
\text { web }\end{array}$ & Whatsapp & Twitter & Facebook & Youtube \\
\hline $\begin{array}{l}\text { En lo } \\
\text { posible }\end{array}$ & 0 & $\begin{array}{c}2 \text { (uso } \\
\text { modal) }\end{array}$ & 0 & 0 & 0 & 0 & 0 & 0 \\
\hline $\begin{array}{l}\text { En la } \\
\text { medida de } \\
\text { lo posible }\end{array}$ & $\begin{array}{c}2 \\
\text { (modales) }\end{array}$ & $3(1 / 2)$ & 1 (1 modal) & 2 (uso 1) & 0 & 0 & 0 & 0 \\
\hline
\end{tabular}

Tabla 11. Presencia de las dos formas en el corpus MEs $A$

\section{CONCLUSIONES}

Las formas consideradas, en lo posible y en la medida de lo posible, aparecen en los corpus con dos valores básicos: estableciendo una limitación a la realización de la acción y con un contenido procedimental ligado a la atenuación de la función ilocutiva. En este último empleo funcionan como operadores argumentativos atenuativos de actos directivos y desarrollan un comportamiento cortés. El contenido de limitación, generalmente empleado en contextos prospectivos, se va acercando a lo procedimental, pero aún no puede afirmarse que estas construcciones sean operadores en todos sus empleos. A diferencia de otros elementos de su paradigma -posiblemente, a ser posible o si es posible-, en lo posible y en la medida de lo posible no dejan en suspenso la aserción sino el grado de realización de la misma. Se han especializado en este mismo contexto, extendiendo el capítulo de los operadores discursivos.

\section{BIBLIOGR AFÍA}

Albelda, Marta y Ana María Cestero (2011): «De nuevo, sobre los procedimientos de atenuación lingüística», Español Actual: Revista de español vivo, 96, pp. 121-155. 
Berrendonner, Alain (1990): «Pour une macro-syntaxe», Travaux de Linguistique, 21, pp. 25-36.

Berrendonner, Alain (2003): «Eléments pour une macro-syntaxe. Actions communicative, types de clauses, structures périodiques», en Antonietta Scarano (ed.), Macro-syntaxe et pragmatique. L'analyse linguistique de l'oral, Roma, Bulzoni, pp. 93-109.

Blanche Benveniste, C. (2002): «Macro-syntaxe et micro-syntaxe: les dispositifs de la rection verbale», en Hanne Leth Andersen y Henning Nolke (eds.), Colloque international. Macro-syntaxe et macro-semantique, Berna, Peter Lang, pp. 95-118.

BRINTON, Laurel J. (2010): «Discourse Markers», en Andreas H. Jucker e Irma Taavitsainen (eds.), Historical Pragmatics, Berlin, De Gruyter Mouton, pp. 285-314.

BRIZ, Antonio (1995): «La atenuación en la conversación coloquial. Una categoría pragmática», en Luis Cortés (ed.), El español coloquial. Actas del I Simposio sobre análisis del discurso oral, Almería, Universidad de Almería, pp. 103-122 [ampliado en Antonio Briz (1998), caps. 4 y 6].

BRIZ, Antonio (1998): El español coloquial en la conversación. Esbozo de pragmagramática, Barcelona, Ariel.

BRIZ, Antonio (2005): «Eficacia, imagen social e imagen de cortesía. Naturaleza de la estrategia atenuadora en la conversación coloquial española», en Diana Bravo (ed.), Estudios de la (des)cortesía en español. Categorías conceptuales y aplicaciones a corpus orales y escritos, Estocolmo-Buenos Aires, Dunken, 51-89.

BRIZ, Antonio (2007): «Para un análisis semántico, pragmático y sociopragmático de la cortesía atenuadora en España y América», LEA: Lingüistica española actual, XXIX, 1, pp. 5-44.

Briz, Antonio, Salvador Pons y José Portolés (coords.) (2008-actualidad): Diccionario departiculas discursivas del español. En línea: < http://www.dpde.es >.

Briz, Antonio y Marta Albelda (2013): «Una propuesta teórica y metodológica para el análisis de la atenuación lingüística en español y portugués. La base de un proyecto en común (ES.POR.ATENUACIÓN)», Onomázein, 28, pp. 288-215.

Buenafuentes, Cristina (2007): Procesos de gramaticalización y lexicalización en la formación de compuestos en español. Tesis doctoral. En línea: <https://ddd. uab.cat/pub/tesis/2007/tdx-0321107-172834/cbm1de1.txt>.

Caffi, Claudia (1999): «On mitigation», Journal of Pragmatics, 31, 7, pp. 881909.

Caffi, Claudia (2007): Mitigation, Oxford, Elsevier. 
Cestero, Ana María y Marta Albelda (2012): «La atenuación lingüística como fenómeno variable», Oralia, 15, pp. 77-124.

Cifuentes Honrubia, J. L. (2003): Locuciones prepositivas. Sobre la gramaticalización preposicional en español, Alicante, Universidad de Alicante.

Claridge, Claudia y Leslie Arnovick (2010): «Pragmaticalisation and Discursisation», en Andreas H. Jucker e Irma Taavitsainen (eds.), Historical Pragmatics, Berlin, De Gruyter Mouton, pp. 165-192.

Company, Concepción (2003): «La gramaticalización en la historia del español», Medievalia, 35, pp. 3-61.

CORDE: Corpus Diacrónico del Español. Real Academia Española.

CORPES: Corpus del Español del siglo XXI. Real Academia Española.

CREA: Corpus de referencia del español actual. Real Academia Española.

Diewald, Gabriele (2011): «Pragmaticalization (Defined) as Grammaticalization of Discourse Functions », Linguistics, 49, 2, pp. 365-390.

Dik, Simon C. (1997): The Theory of Functional Grammar II. Complex and Derived Constructions, Berlin/New York, Mouton de Gruyter.

Dostie, Gaetane (2004): Pragmaticalisation et marqueurs discursifs, Bruxelles, De Boeck-Duculot.

Douglas De Sirgo, Silvina (2007): Estrategias discursivas de la atenuación en Tucumán, Tucumán, Universidad Nacional de Tucumán [colección Tesis].

Ducrot, Oswald (1995): «Les modificateurs déréalisants», Journal of Pragmatics, 24, pp. 145-165.

ERman, Britt y Ulla-Britt Kotsinas (1993): «Pragmaticalization: The Case of ba' and you know», Studies I modern sprakvetenskap [Acta Universitatis Stockholmiensis, New Series 10], Stockholm, Almqvist and Wiksell, pp. 79-93.

FÉLIX-BRASDEFER, César (2004): «La mitigación en el discurso oral de mexicanos y aprendices de español como lengua extranjera », en Diana Bravo y Antonio Briz (eds.), Pragmática sociocultural: estudios sobre el discurso de cortesía en español, Barcelona, Ariel, pp. 285-299.

FRASER, Bruce (1980): «Conversational mitigation », Journal of Pragmatics, IV, 4, pp. 341-350.

FRASER, Bruce (2010): «Pragmatic Competence: The Case of Hedging», en Gunther Kaltenböck, Wiltrud Mihatsch y Stefan Schneider (eds.), New approaches to Hedging, Bingley, Emerald Publishing, pp. 15-34.

Fuentes Rodríguez, Catalina (1996): La sintaxis de los relacionantes supraoracionales, Madrid, Arco Libros.

Fuentes Rodríguez, Catalina (2003): «Operador/ conector, un criterio para la sintaxis discursiva », Rilce. Revista de Filología Hispánica, 19, 1, pp. 61-85. 
Fuentes Rodríguez, Catalina (2007): La sintaxis del enunciado: los complementos periféricos, Madrid, Arco Libros.

Fuentes Rodríguez, Catalina (2010): La gramática de la cortesía en español/LE, Madrid, Arco Libros.

Fuentes Rodríguez, Catalina (2016): «Para colmo, scalar operator and additive connector. Keys to an evolving process », Journal of Historical Pragmatics, 17,1, pp. 79-106.

Fuentes Rodríguez, Catalina (2017a[2000]): Lingüistica pragmática y análisis del discurso, Madrid, Arco Libros, 3. ${ }^{\mathrm{a}}$ ed.

Fuentes Rodríguez, Catalina (2017b): «Macrosintaxis y lingüística pragmática», Círculo de Lingüistica Aplicada a la Comunicación (CLAC), 71, pp. 5-34.

Fuentes Rodríguez, Catalina (2018[2009]): Diccionario de conectores y operadores del español, Madrid, Arco Libros.

Fuentes Rodríguez, Catalina (2019): «Categorías discursivas y segmentación en macrosintaxis», en Catalina Fuentes y Salvador Gutiérrez (eds.), Avances en macrosintaxis, Madrid, Arco Libros, pp. 15-65.

Garachana, Mar (1999): «Los procesos de gramaticalización», Moenia, 5, pp. 155- 172.

Haverkate, Henk (1994): La cortesía verbal. Estudio pragmalingüístico, Madrid, Gredos.

HeIne, Bernd (2003): «Grammaticalization», en Brian D. Joseph y Richard D. Janda (eds.), The handbook of historical linguistics, Malden, Blackwell, pp. 575601.

HeIne, Bernd (2013): «On discourse markers: Grammaticalization, pragmaticalization, or something else? », Linguistics, 51, 6, pp. 1205-1247.

Hopper, Paul J. (1991): «On some principles of grammaticization», en Elizabeth C. Traugott y Bernd Heine (eds.), Approaches to grammaticalization, Amsterdam, John Benjamins, pp. 17-35.

Jørgensen, Annette M. (2009): «"En plan” used as a hedge in Spanish teenage language», en Anna-Brita Stenstrøm y Annette Myre Jørgensen (eds.), Youngspeak in a multilingual perspective, Amsterdam, John Benjamins P.C., pp. 95-119.

Kaltenböck, Gunther, Wiltrud Minatsch y Stefan Schneider (eds.) (2010): New Approaches to Hedging, Studies in Pragmatics, United Kingdom, Emerald Group Publishing.

Kaltenböck, Gunther, Bernd Heine y Tania Kuteva (2011): «On thetical grammar», Studies in Language, 35, 4, pp. 852-897.

Lehmann, Christian (1982): Thoughts on Grammaticalization: A Programmatic Sketch, I, Köln, Universidad de Köln. 
López-Couso, María J. (2010): «Subjectification and Intersubjectification», en Andreas H. Jucker e Irma Taavitsainen (eds.), Historical Pragmatics, Amsterdam, De Gruyter Mouton, pp. 127-163.

Meyer-Hermann, Reinhard (1988): «Atenuación e intensificación (análisis pragmático de sus formas y funciones en español hablado)», Anuario de Estudios Filológicos, XI, pp. 275-290.

Montes Giraldo, José Joaquín (1980-81): «Sobre el como de atenuación», Boletin de Filologia, vol. 31, pp. 667-675.

Nuyts, Jan (2001): Epistemic Modality, Language and Conceptualization: A Cognitive-Pragmatic Perspective, Amsterdam, John Benjamins.

Puga LaRraín, Juana (1997): La atenuación en el castellano de Chile: un estudio pragmalingüistico, [Tesis] València, Universitat de València.

Samper Hernández, Marta (2011): «Estrategias de atenuación en el español de Las Palmas de Gran Canaria», comunicación presentada en el XVI Congreso Internacional de la Alfal, Alcalá de Henares, 6-9 de junio de 2011.

Santos Río, Luis (2003): Diccionario de partículas, Salamanca, Luso Española de Ediciones.

SBISÁ, Marina (2001): «Illocutionary force and degrees of strength in language use», Journal of Pragmatics, 33, 12, pp. 1791-1814.

SCHNeIDER, Stefan (2013): «La atenuación gramatical y léxica», Oralia: Análisis del discurso oral, 16, pp. 335-358.

Stein, Dieter y Susan Wright (eds.) (1995): Subjectivity and Subjectivisation, Cambridge, Cambridge University Press.

Traugott, Elizabeth C. (1995): «The Role of the Development of Discourse Markers in a Theory of Grammaticalization », Paper presented at ICHL XII, Manchester. En línea: <http://www.stanford.edu/ traugott/papers/discourse.pdf> [10/06/2014].

Traugott, Elizabeth C. (2003): «Constructions in Grammaticalization», en Brian D. Joseph y Richard D. Janda (eds.), Handbook of Historical Linguistics, Blackwell, Londres, 624-647.

Fecha de recepción: 6 de julio de 2019 Fecha de aceptación: 25 de octubre de 2019 
